\title{
Fetal therapy for surgical abnormalities the fetus as a patient
}

\begin{abstract}
Fetal surgery is the act of opening the gravid uterus, surgically correcting a fetal abnormality, and returning the fetus to the uterus for postoperative recovery and continued gestational development. This article discusses the main advances in fetal surgical therapy aiming to inform health care professionals about the state-of-the-art techniques and future challenges in this field. UCSF Surgeon Reflects on Performing World's First Fetal Surgery 30Years Ago. Fetal therapy is a logical extension of fetal diagnosis. A group of disorders amenable to potential improvement by fetal treatment has been identified, including fetal urinary tract obstruction, fetal diaphragmatic hernia, fetal congenital cystic adenomatoid malformation, fetal sacrococcygeal teratoma, Myelomeningocele, Cardiac ventricular outflow obstruction,and Cerebrospinal fluid obstruction. The discovery of effective tocolysis would be analogous to the development of effective immunosuppression and would allow fetal surgery to achieve its full potential. The fetal surgical experience with each of these lesions is reviewed and the maternal risk of fetal surgery is discussed in the article. The aim of the review is to identify the effectiveness of maternal-fetal surgery for several congenital abnormalities \& surgeries of greatest interest including open fetal surgery and fetoscopic surgery and their comparison to post natal surgeries.
\end{abstract}

Keywords: fetal surgery, fetal therapy, fetoscopic surgery, fetal ultrasonography, ultrasound, tocolysis, congenital abnormalities
Volume 4 Issue I - 2018

\author{
Sanjaya INH, Suwardewa TGA, Kusuma \\ AANJ, Negara IKS, Putra IWA, Wiradnyana \\ AAP,Widiyanti ES, Mulyana RS, Pangkahila ES, \\ Florencia Desiree \\ Obsterics and Gynecology Department of Udayana University, \\ Feto-Maternal Division, Sanglah Hospital, Indonesia
}

\author{
Correspondence: Pangkahila ES, Obsterics and Gynecology \\ Department of Udayana University, Feto-Maternal Division, \\ Sanglah Hospital, Indonesia,
}

Email florenciadesiree88@gmail.com

Received: August 31, 2017| Published: January 31, 2018

\section{Introduction}

Historically, the gold standard for the treatment of congenital malformations has been planned delivery at tertiary care center with attempted post-natal repair or amelioration of the lesion. Over the last few decades however, rapid advances in imaging and instrumentation technology combined with superior knowledge of fetal pathophysiology has led to the development of novel intrauterine interventions for most common fetal anomalies and to save the fetus life.

Surgical intervention in the fetus should minimize the risk to the mother's life and the future of her fertility. Advances in fetal therapy would have been impossible without the preceding advancements in imaging that have taken place over the last 40years. The development of prenatal ultrasound (US) in the 1970s, as the use of three-dimensional ultrasound and realtime (4D), and MRI with rapid acquisition, all that brought rapid advances in fetal imaging and sampling techniques, allowing for better understanding of fetal pathophysiology, this contributes greatly to the development of fetal surgery. It improves the accuracy in determining fetal abnormalities as early as possible in the first trimester. Recent developments in maternal anesthesia, tocolytics, and technical aspects of surgery also play a role in reducing risks to the fetus and mother. ${ }^{1,2}$

Correction of anatomical malformations in utero with open fetal surgery has risks in pregnancy for both mother and fetus. Some of the risks of surgery, anesthesia, and preterm labor cause the open fetal surgery to be very limited only under certain conditions ie in the disorder that will result in permanent damage and if the intervention will provide normal development, then this intervention can be done. Fetal surgery is performed on a limited number of institutions requiring the following skills: ${ }^{3}$
A. Fetomaternal specialists who have experience on prenatal intervention.

B. Sonographer who has extensive experience and ability on fetal diagnosis.

C. A child surgeon who has experience performing surgery on preterm babies with small size.

D. An environment or work unit that has a high risk obstetric unit that is associated with tertiary intensive care.

E. A team of specialists including geneticists, anesthesiologists, neonatologists, urologists, neurosurgeons, cardiologists, radiologists who work together well in counseling and intervention processes.

F. A high standard environment overseen by a regulatory body and a fetal surgeon committee composed of peers not involved in surgery and a qualified ethicist.

G. A clinical pathologist who is capable of performing highstandard examinations and has performed an examination on an adequate number of fetal models.

Malformations diagnosed and will be performed fetal surgery must meet the following criteria: ${ }^{4}$

A. Prenatal diagnostic techniques should identify the malformation and exclude other lethal malformations with a high degree of certainty.

B. The defect should have a defined natural history and cause progressive injury to the fetus that is irreversible.

C. Repair of the defect should be feasible and should reverse or prevent the injury process. 
D. Surgical repair must not entail excessive risk to the mother or her future fertility

There is a list of anatomical abnormalities that can theoretically provide satisfactory results with open fetal surgery (Table 1). This discussion is limited to abnormalities that have actually shown encouraging results in which fetal surgery has been performed.

\section{Open fetal surgery in the future}

A major challenge in open fetal surgery is to minimize the risks to the mother and fetus. Unfortunately, foetal morbidity and mortality rates remain excessively high after such interventions, due to (a) direct operative trauma to the myometrium, foetal membranes and foetus, and (b) effects of postoperative premature membrane rupture, uterine flow decrease and the increase of uterine contractions. The latter are almost always observed after foetal surgery and may lead to premature labour and foetal demise. Although the vast majority of malformations detected in the foetus are managed best after birth (Table 1), certain cases lead to irreversible damage if not prenatally corrected.

The inherent characteristics of foetal wound healing, which is scarless at mid gestation, in contrast to wound healing in adults. Prenatal correction of cleft lip and palatum clefts or other abnormalities gives the advantage of healing the wound without scarring, which is possible because of biological processes in the fetus and better remodeling process. When new surgical techniques are used, and supported by a good work system and adequate tocolycic therapy, it can reduce the risk of both the fetus and the mother the conditions and indications for surgery in the fetus will be more possible.,

Table I Indication for fetal intervention

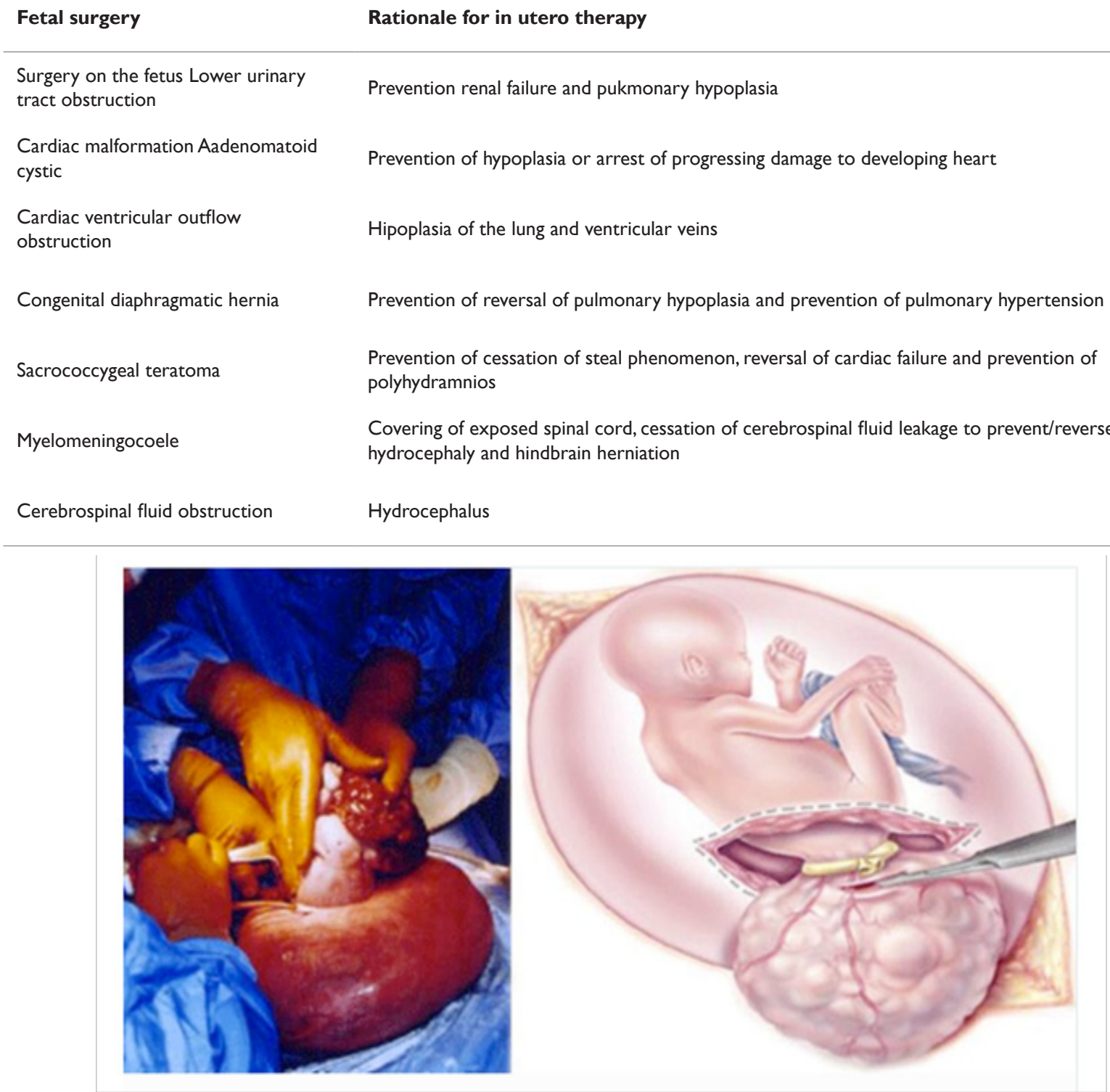

Figure I Open fetal surgery with sacchosgial teratoma. ${ }^{6}$ 
Table 2 Fetal intervention with endoscopy. ${ }^{3}$

Indication (Potention)
Separation of Twins
Ligation of the umbilical cord
Laser urethral posterior laser ablation
Tracheal occlusion
Ablation or embolization of the tumor in the fetus
Patching the membrane rupture
Closure of the spine bifida defect

Hematopoietic stem cell transplantation and gene therapy in the intra uterine fetus

\section{Fetoscopy for fetal abnormalities}

With the rapid advancement of laparoscopic techniques resulting in the discovery of technologists that allow fetoscopic procedures to be performed and the ability to perform major surgery of the fetus with direct vision through a small puncture hole in the uterus has become a reality. A large number of procedures have been and will be done in the future using the technique of fetoscopy (Table 2) that may decrease maternal and fetal risk and risk of preterm delivery.

Fetoscopy is a procedure that utilizes an instrument called a fetoscope to evaluate or treat the fetus during pregnancy. Currently, the most common fetoscopy procedure is not performed on the fetus, such as laser ablation in vascular anastomosis for twin-twin transfusion syndrome and umbilical cord ligation or performing both procedures in the case of acardiac twins as well as in complicated monochorionic twin pregnancies. Procedures in the fetus, such as fetal cystoscopy with laser ablation of the posterior urethral valve, are now technically feasible, but have not received clinically encouraging results. With recent advances in laparoscopic equipment and techniques, minimally invasive clinical applications for fetal surgery will increase. However, the fetoscopic procedure is still at risk significantly related to the size and quantity of trokar used and determined also by the severity of the fetal abnormalities treated. ${ }^{3}$

The most effective percutaneous uterine fetal surgery has been performed for both evaluation and therapeutic purposes in obstructive uropathy. Lower urinary tract obstruction (LUTO) is a heterogeneous condition that can affect 1 in 5000 or 1 of 8000 baby boys. Posterior urethral valve (PUV) or urethral atresia is the most common cause of LUTO, but other cause such as urethral meatus stenosis, anterior urethral valve, ureteric ectopic insertion and tumors of the bladder have been found. ${ }^{5}$

\section{Maternal, fetal and risk management}

Fetal surgery is a unique procedure because fetal surgery is still associated with high risks for the mother and the fetus, including both morbidity of surgical procedures and the possibility of future pregnancies. Maternal safety is the most important in fetal surgical procedures, management in maternal and fetal service units are interdependent. Before being applied to humans, it is important to develop anesthesia, surgery and tocolytic techniques for maternal safety by taking experiments on animals. Researchers are now continuing to develop ways to improve maternal and fetal safety and improve clinical outcomes in about 400 cases of open fetal surgery performed. ${ }^{4}$ Wilson et al used the term matemal-fetal surgery for fetal surgery. Because surgery done on the mother and also in the fetus. Fetal surgery also leads to enhanced surgical and anaesthetic risk in the mother including haemorrhage, infection, airway difficulties and amniotic fluid embolism, premature rupture of membranes during preterm, preterm delivery, drug side effects (tocolytics), elongation hospital care, fertility impairment, recurrent cesarean section, and death. Risks to the fetus are surgical trauma, prematurity, drug side effects and fetal or neonatal death. ${ }^{7}$

Albanese et al reported three fetal cases with open fetal surgery sakrokoksigeal teratoma. One fetus died on the first postoperative day which may be due to hyperkalemia because of large-size tumor necrosis. The second fetus obtained good results and was born at the age of 29 weeks and followed by postnatal surgery. In the third fetus lasts for 7 weeks after open fetal surgery. ${ }^{3}$

\section{Operation technique}

Before open fetal surgery was performed, the mother was given $50 \mathrm{mg}$ of indomethacin suppository and then performed an epidural catheter. After induction of general anesthesia with isoflurane, then the mother is placed in supine position with her right side higher to avoid suppression of uterine vessels. Infusion fluid and foley catheters were monitored during surgery and postoperatively. ${ }^{4}$

The operator made a large incision in the lower abdominal. For most patients, the incision on the fascia is done in the midline to get a wider space. If the placenta is anterior then a posterior hysterotomy is needed, rectal muscle separation may be required. Surgical field can be expanded using retractors. The isoflurane anesthetic level is given by titration to achieve adequate uterine relaxation before the uterine incision. Tocolytic preparations that should be provided in the operating room are magnesium sulphate, terbutaline and nitroglycerine, which are used selectively to control uterine contractions. ${ }^{4}$

When the location of the hysterotomy has been determined, the clinician maps the placental boundary and notes the edge of the placenta using ultrasound and marks the uterine surface with an electrocautery. Hysterotomy will be performed at least $5 \mathrm{~cm}$ from the edge of the placenta and as far as possible. A uterine wire piercing that has been modified and burned (cauterized) when inserted into the uterus. ${ }^{4}$

Fetal heart rate is monitored with ultrasound while fetal heart function is monitored by fetal pulse oximetry. The fetus is kept warm by limiting exposure and continuously irrigate ringer lactate fluid into the amniotic cavity. Anesthesia also given to the fetus by administering intramuscular anesthetic injections and intramuscular medications. Fetal intravenous access is performed through intravenous peripheral when necessary. After tthe surgery to the fetus is complete, the fetus is put back into the uterine cavity, and the amniotic fluid is replaced with a warm ringer lactat containing antibiotics. The uterus is covered with two layers of stitches with absorbable suture material and covered by omentum to prevent amniotic fluid leakage.

Postoperative period, the mother is monitored in the ICU room. The tocolytic therapy used is magnesium sulphate, indomethacin and terbutaline may be added if necessary. Fetal activity and fetal heart rate are continuously monitored with tocodynamometry and fetal well-being is evaluated twice daily with ultrasound and oncedaily fetal electrochardiography. Epidural catheters are an important component used to reduce the stress response in the mother and the 
incidence of preterm labor. After the second postoperative day, the mother was transferred to the treatment room. Usually allowed to go home on the fourth day. ${ }^{4}$

\section{Maternal output}

In more than 400 open fetal surgeries, no maternal deaths and major maternal complications were found. The most notable complication is non-cardiac pulmonary edema. This only happens in two cases. This is a manifestation of matemal mirror syndrome which occurs before surgery and then required termination of tocolytic administration and gave birth to a fetus that has not been viable. In three cases, this happened without any apparent reason. Ventilation needs to be given before repair. Another complication is the release of the amniotic membrane with the occurrence of amniotic fluid leakage, pseudomembranous colitis, and wound complications. This should be known to the mother before surgery is performed and the pregnancy will then be delivered with sectio cesarea because of the location of the hysterotomy incision made in the upper uterus during fetal surgery. ${ }^{4,7}$

\section{Conclusion}

Prenatal diagnosis dramatically alters the clinical perception of the fetus. The fetus is now worth mentioning as a patient, and fetal therapy against so many types of diagnosed disorders when prenatal can be offered to do. Medical treatment for the fetus is now standard treatment for a number of disorders and provides significant therapeutic benefits. Many benefits are gained for various medical diseases and are proven to be treatable but the results are imperfect and further research and clinical experience is needed. The more invasive the therapeutic maneuver the more significant the risks to the mother and fetus, as well as raising difficult questions about the rights that the fetus has, the risks and benefits. Because of the high risk of open fetal surgery, it is important to conduct a controlled clinical trial..$^{3-5,7-9}$

Medical innovations, especially surgical interventions, sometimes occur unplanned where the intervention developing into a standard of treatment without adequate scientific and ethical evaluation. Fetal surgery is one example where this unmanaged innovation has had an impact on many patients. The latest innovation in fetal surgery for spina bifida abnormalities, a relatively frequent disorder and usually diagnosed in the second trimester, increases the chances of fetal surgery in more patients. This development challenges the medical community to guide this ongoing innovation within the framework of ethical responsibility, where extensive support from the professional community is required. The transition from innovation to standard of medical services for fetal surgery should be guided by adequate ethical guidelines. ${ }^{7}$

\section{Acknowledgements}

None.

\section{Conflict of interest}

Author declares that there is no conflict of interest.

\section{References}

1. Arca MJ, Teich S. Current controversies in pennatal care: fetal versus neonatal surgery. Clin Perinatol. 2004;31(3):629-648.

2. Wilson RD, Johnson MP, Flake AW, et al. Reproductive outcomes after pregnancy complicated by matemal-fetai surgery. Am J Obstet Gynecol. 2004;191(4):1430-1436.

3. Albenese CT, Harrison MR, Paek B, et al. Surgical therapy for fetal anornalies. In: Callen PW. editors. Ultrasonograpy in Obstetrics and Gynecology. 4th ed. USA: W.B. Saunders company; 2000. p. 763-780.

4. Flake AW. Fetal therapy. Medical and surgical approaches. In: Creasy RK, Resnik R, editors. Matemal fetal medicine, Principles and practice. 5th ed. Philadelphia, Pennsylvania: Elsevier; 2004. p. 483-494.

5. Gabbe. Open Fetal Surgery. Obstetrics - Normal and Problem Pregnan cies. 4th ed. UK: Churchill Livingstone Inc; 2002. p. 303-312.

6. http://www.chop.edu/treatments/fetal-surgery-sacrococcygeal-teratoma-sct/about\#.V-4xsemD7ow.'

7. Hirose S, Farmer OL. Fetal surgery for sacrococcygeal teratoma. Clin Perinatol. 2003;30(3):493-506.

8. Chervenak FA, McCullough LB. A comprehensive ethical framework for fetus research and its application to fetal surgery for spina bifida. Am J Obstet Gynecol. 2002;187(1):10-14.

9. Bruner JP. Matemal-fetal surgery. Clin Perinatol. 2003;30(3):xiii-xvi. 